\title{
Risk stratification protocol to reduce consumption of personal protective equipment for emergency surgeries during COVID-19 pandemic
} \author{
Charmaine KL Chu ${ }^{3}$, FHKAM (Anaesthesiology), Sandy KY Chau ${ }^{4}$, FHKAM (Pathology), TL Chow 5 , FHKAM (Surgery), \\ Huey S Lim³ ${ }^{3}$ FHKAM (Anaesthesiology), Kitty SC Fung ${ }^{4}$, FHKAM (Pathology), \\ Kenneth KW Li ${ }^{1,2}$ *, FRCOphth, FHKAM (Ophthalmology) \\ ${ }^{1}$ Department of Ophthalmology, United Christian Hospital, Hong Kong \\ ${ }^{2}$ Department of Ophthalmology, Tseung Kwan O Hospital, Hong Kong \\ ${ }^{3}$ Department of Anaesthesiology, United Christian Hospital, Hong Kong \\ ${ }^{4}$ Department of Pathology, United Christian Hospital, Hong Kong \\ ${ }^{5}$ Department of Surgery, United Christian Hospital, Hong Kong \\ *Corresponding author: kennethli@rcsed.ac.uk
}

Daniel HT Wong 1,2, FRCOphth, Emily WH Tang1,2, FHKAM (Ophthalmology), Anthony Njo33, FHKAM (Anaesthesiology),

Hong Kong Med J 2020;26:252-4

https://doi.org/10.12809/hkmj208533

This article was published on 5 May 2020 at www.hkmj.org.
The World Health Organization declared coronavirus disease 2019 (COVID-19) a global pandemic on 11 March 2020. Although many patients with severe acute respiratory syndrome coronavirus 2 (SARS-CoV-2) infection develop respiratory symptoms, transmission of the virus has been reported to occur during the asymptomatic phase. ${ }^{1,2}$ In symptomatic patients, computed tomography of thorax shows characteristic lung findings; however, polymerase chain reaction (PCR) test is required to confirm the diagnosis, and this is also effective in asymptomatic patients. The main modes of SARS-CoV-2 transmission include respiratory droplets, physical contact, and aerosol generating procedures (AGP). ${ }^{2,3}$ There is currently no evidence confirming airborne transmission of SARS-CoV-2. ${ }^{3}$

During the 2002-3 SARS outbreak, 21\% of infected cases were healthcare workers. ${ }^{4,5}$ Aerosol generating procedures, such as intubation and airway aspiration, are routine procedures during general anaesthesia. The appropriate use of personal protective equipment (PPE) during AGP, including N95 respirator, face shield or goggles, disposable gloves, splash-resistant gown (full PPE) effectively protects healthcare workers against SARS-CoV-2 infection. ${ }^{6,7}$ Surgical procedures involving patients with suspected COVID-19 should also be performed in an airborne isolation room with negative atmospheric pressure. ${ }^{6}$ In emergency situations, any delay in intervention caused by lack of clear protocol can increase morbidity and mortality of patients. Indiscriminate or universal PCR testing for all such patients is not justified, as it is time-consuming, resource-draining, and may not be feasible in emergency settings.

We developed a risk stratification protocol for all emergency surgeries, with two main principles:
(1) to provide a framework for healthcare workers to make timely assessments and decisions for emergency surgeries; and (2) to provide adequate PPE and promote appropriate use of PPE, especially during AGP (Fig). ${ }^{8}$ Consensus of the protocol was achieved with all stakeholders, including specialists in infection control, anaesthesiology, emergency medicine, and all surgical streams in our institution, including general surgery, obstetrics and gynaecology, ophthalmology, orthopaedics, and otorhinolaryngology. Patients were stratified according to epidemiological history screening by FTOCC (fever, travel, occupation, contact, and clustering) and upper respiratory tract symptoms in the context of fever. Positive travel history was defined as any travel to outbreak areas within 2 weeks prior to presentation. ${ }^{9}$ Patients were stratified as high risk if they were positive either on FTOCC screening or for upper respiratory tract symptoms. While awaiting surgery, high-risk patients underwent nasopharyngeal and throat swabs for PCR tests, and results were returned within 4 hours. In overriding emergency cases, surgery was performed without waiting for the PCR test results. Overriding emergency cases and patients who had positive PCR test results for SARS-CoV-2 infection required the use of eight sets of full PPE (two for anaesthetist, one for operation room assistant, two for runner nurses, one for scrub nurse, two for surgeon) and an airborne isolation room with negative pressure. For patients who had negative PCR test results, standard precautions were adopted, including surgical gown, sterile gloves, standard surgical mask, and cap for all members of the surgical team, and two sets of full PPE for the anaesthetist and operation room assistant when necessary.

We conducted a retrospective analysis of the 
consumption of PPE before (2-8 February 2020) and after (1-7 March 2020) the implementation of our risk stratification protocol. Before implementation of our protocol, five of 59 patients would have been considered high risk (one laparoscopic salpingectomy for ruptured ectopic pregnancy, one emergency repair of paediatric limb fracture, one emergency tracheostomy, one emergency caesarean section, and one urgent repair of injury to femoral artery). Of them, four underwent surgery in an isolation operation room and 32 sets of full PPE were used. After implementation of our protocol, 14 of 49 patients were identified as high risk; however, only two of the 14 patients were overriding emergency cases (one emergency caesarean section and one laparoscopic cholecystectomy), and 16 sets of full PPE were used. All PCR test results for SARS-CoV-2 were negative during the study period before and after implementation of our protocol. No healthcare workers were infected. There was a significant reduction in the proportion of high-risk cases requiring full PPE after the introduction of our new protocol $(\mathrm{P}=0.017$, Fisher's exact test). A total of 72 sets of PPE were saved after implementation of our protocol.

Our protocol provides a clear framework for all frontline healthcare workers across different disciplines, avoiding delay in management of emergency surgical conditions. It can also provide adequate and appropriate protection of all healthcare workers during the COVID-19 outbreak. Amid the global pandemic, many countries are facing shortages of PPE, and the World Health Organization has called on the industry to increase manufacturing to meet the rising demand. ${ }^{10}$ Conservation of PPE is crucial.

\section{Author contributions}

Concept or design of the study: EWH Tang and A Njo.

Acquisition of data: All authors.

Analysis or interpretation of data: All authors.

Drafting of manuscript: DHT Wong, EWH Tang and KKW Li. Critical revision of the manuscript for important intellectual content: All authors.

All authors had full access to the data, contributed to the study, approved the final version for publication, and take responsibility for its accuracy and integrity.

\section{Conflicts of interest}

As an editor of the journal, Kenneth KW Li was not involved in the peer review process. Other authors declare no competing interest.

\section{Acknowledgement}

We acknowledge all Chiefs of Service of all clinical departments in United Christian Hospitals for their approval of the workflow, and Department of Pathology for performing the tests.

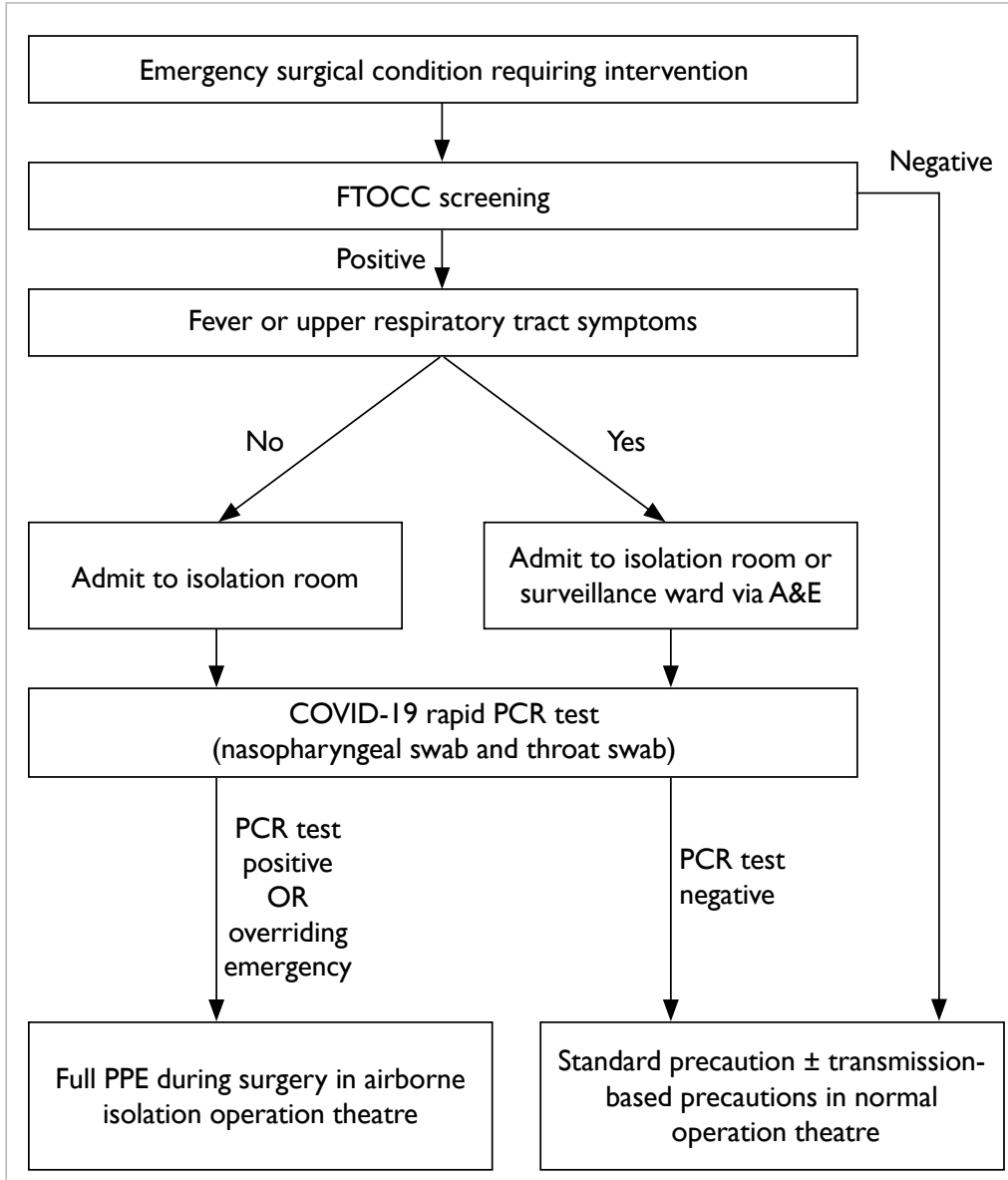

FIG. Risk stratification protocol for arrangement of emergency operation Abbreviations: A\&E = accident and emergency; COVID-19 = coronavirus disease $2019 ; \mathrm{FTOCC}=$ fever, travel history to areas with active community transmission of COVID- 19 within the past 14 days, occupation, contact, and clustering; Full PPE = full personal protective equipment, including splash resistant gown, disposable gloves, N95 respirator, and goggles or face shield; $P C R=$ polymerase chain reaction

\section{Funding/support}

This commentary received no specific grant from any funding agency.

\section{Ethics approval}

The study was conducted in accordance with the Declaration of Helsinki. The patients provided informed consent for all procedures.

\section{References}

1. Bai Y, Yao L, Wei T, et al. Presumed asymptomatic carrier transmission of COVID-19. JAMA 2020 Feb 21. Epub ahead of print.

2. Centers for Disease Control and Prevention, US Department of Health \& Human Services. Symptoms of coronavirus disease 2019 (COVID-19). Available from: https://www.cdc.gov/coronavirus/2019-ncov/about/ symptoms.html. Accessed 28 Mar 2020.

3. World Health Organization. Modes of transmission of 
virus causing COVID-19: implications for IPC precaution recommendations. Available from: https://www.who.int/ news-room/commentaries/detail/modes-of-transmissionof-virus-causing-covid-19-implications-for-ipcprecaution-recommendations. Accessed 28 Mar 2020.

4. World Health Organization. Summary of probable SARS cases with onset of illness from 1 November 2002 to 31 July 2003. Available from: https://www.who.int/csr/sars/ country/table2004_04_21/en. Accessed 28 Mar 2020.

5. Caputo KM, Byrick R, Chapman MG, Orser BJ, Orser BA. Intubation of SARS patients: infection and perspectives of healthcare workers. Can J Anaesth 2006;53:122-9.

6. Centers for Disease Control and Prevention, US Department of Health \& Human Services. Coronavirus disease 2019 (COVID-19). Infection control guidance. Available from: https://www.cdc.gov/coronavirus/2019ncov/infection-control/control-recommendations.html. Accessed 28 Mar 2020.

7. European Centre for Disease Prevention and Control. ECDC technical report. Infection prevention and control for COVID-19 in healthcare settings-first update. 12 March 2020. Available from: https://www.ecdc.europa. eu/sites/default/files/documents/COVID-19-infectionprevention-and-control-healthcare-settings-march-2020. pdf. Accessed 28 Mar 2020.

8. Lai TH, Tang EW, Chau SK, Fung KS, KK Li. Stepping up infection control measures in ophthalmology during the novel coronavirus outbreak: an experience from Hong Kong. Graefes Arch Clin Exp Ophthalmol 2020 Mar 3. Epub ahead of print.

9. Health notice, Coronavirus disease 2019 (COVID-19). Centre for Health Protection, Department of Health, Hong Kong SAR Government. Available from: https://www.chp. gov.hk/en/index.html. Accessed 26 Mar 2020.

10. Shortage of personal protective equipment endangering health workers worldwide. World Health Organization. Available from: https://www.who.int/news-room/ detail/03-03-2020-shortage-of-personal-protectiveequipment-endangering-health-workers-worldwide. Accessed 28 Mar 2020. 